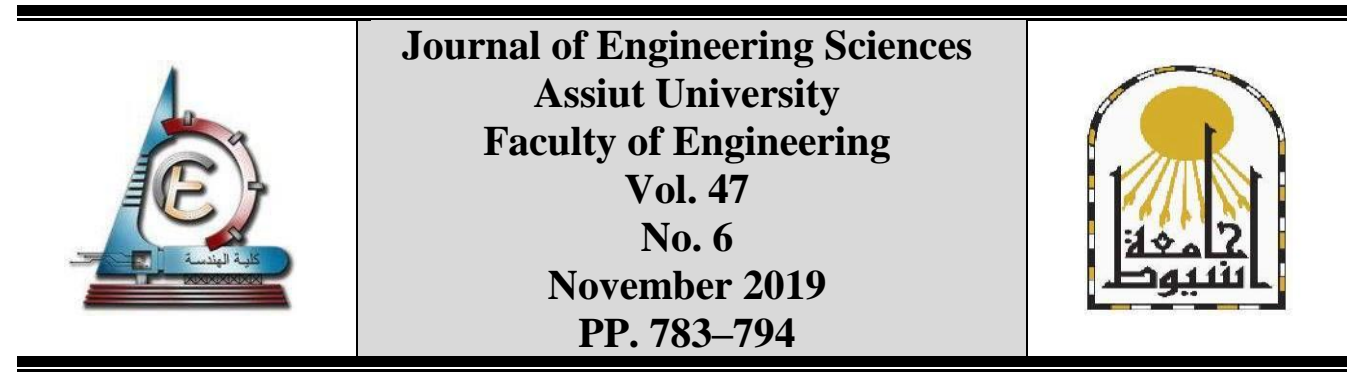

\title{
A NOVEL TECHNIQUE TO MONITOR MEDICAL EQUIPMENT MODES OF OPERATION
}

\author{
E. E. Naguib * and S. A. Fawzi \\ Systems and Biomedical Engineering Department, Faculty of Engineering, Cairo \\ University, Cairo, Egypt.
}

Received 17 June 2019; Accepted 6 July 2019

\begin{abstract}
Quantitative measurement of the effective usability of the medical equipment is an important parameter in the quality and performance assessment. This paper introduces a simple non-invasive technique for real time monitoring of medical equipment modes of operation based on its power consumption pattern. Mode of operation detection is needed to specify the medical equipment reliability, availability, maintainability and usability. The Electrical Signature Analysis technique (ESA), is applied to monitor the overall power consumption of the electromechanical and power components inside the medical equipment.

ESA is extracted using the Root Mean Square (RMS) of the electric current measured by a meter interfaced to a PC via its USB port. The ESA of the medial equipment is recorded, analyzed and correlated with the stored electric current consumption patterns of each specific mode of operation. The results were promising to accomplish the medical equipment monitoring application.
\end{abstract}

Keywords: Clinical engineering, asset management, Medical equipment monitoring, RAM, Usability.

\section{Introduction}

Medical equipment represents an important factor in the growth of healthcare expenditures that requires the efficient management to indicate its proper performance and to ensure its effective usage. The asset management systems were developed to manipulate all the issues related to medical equipment such as calibration, maintenance, repair, user training efficiency and performance [1-3].

The medical equipment mode of operation monitoring may lead to an early detection of malfunction or inefficient operation [4]. The ability to measure real time events is essential to implement data driven-decision systems $[5,6]$.The decision about the medical equipment efficiency and quality of performance should be based upon the resulting parameters such as Reliability, Availability, and Maintainability (RAM). Reliability is defined as the probability that equipment will perform its intended function for a specified

* Corresponding author.

Email address: essam_een@yahoo.com 
period of time [7]. Moreover, Reliability Centered Maintenance (RCM) sets a priority of the equipment' maintenance need to keep its functions by studying the failure modes, sideeffects and risks in order to increase the asset availability, reliability and maintainability instead of the equipment as a physical asset regardless the quality of its functions [8]. The availability is a measure of equipment performance to meet its objectives [9].

$$
\text { Equipment availability }=\frac{\text { Loading time }}{\text { Loading time }- \text { Down time }}
$$

Where, the loading time is the time planned for performing operations and downtime is the time during which the system is not operating [10].

The maintainability according to a periodic plan that guarantees maintenance actions the lifecycle [11]. The management of an efficient of medical equipment requires documents and Computerized Maintenance Management System (CMMS) for testing, inspection, activities, performance monitoring of the equipment, [12 - 13]. The maintenance expenses represent a huge part of an asset management costs; therefore, a reduction in these costs can represent significant saving to the business [14].

One of the evolving practical strategies is the Condition Monitoring Systems (CMS) that monitors the electromechanical components and ensure rising rate of system availability, reliability and productivity in a sustainable manner [15-16]. Condition Based Maintenance (CBM) works better for electromechanical equipment that fails gradually, it is a part of the Condition Monitoring Systems (CMS) strategy, it involves monitoring the equipment status using sensors to measure physical variables such as vibration, sound, energy consumption and torque, which are associated with the equipment performance. $\mathrm{CBM}$ is defined as an automatic maintenance that has the ability to make decisions based on monitoring information or provided by the central control system [17 - 18].

The Electrical Signature Analysis (ESA) is an effective non-invasive technique supporting predictive maintenance and Condition Monitoring (CM) system for rotating electrical machines to improve the reliability of the maintenance strategy [19-20].

S. Zarabi designed a non-invasive system to monitor the real-time electrical consumption with in residential and commercial buildings using an electric current sensor [21]. Sa executed an experiment to detect replacement conditions of railway point machines using electric current shape analysis data for both before and after railway replacement that detect differences between two cases; replace it or not replace it [22]. Yui Ishida invented relates an electric current measuring device by a current transformer attached to the power line to measure an electric current through this power line to control, record, and measure the electric current of measuring equipment [23]. This idea has been already in patent that proved to diagnose the health of a motorized system by measuring vibration, pressure, current, speed, and/or temperature from sensors operatively connected to the motorized system [24].

There are two methods to collect data log about the medical equipment inside hospitals. The first method is the manual data collection that requires extra efforts and time waste. The second method is the automatic data collection via interfacing built-in ports inside equipment with costly and data limited interfacing software for a single manufacturer producing a certain equipment name with specific type and model. 
E. E. Naguib and S. A. Fawzi, A novel technique to monitor medical equipment modes of .........

The proposed approach presents an external monitoring technique to monitor the modes of operation for the medical equipment using the ESA approach. Each operating mode in real time and this electric current meter is connected to a computer for data analysis. This paper introduces the results of monitoring and detecting modes of operation for different types of medical equipment used inside hospitals by a commercial ammeter gauge.

\section{Methodology}

The medical equipment consists of many internal units $\left(U_{1}\right.$ to $\left.U_{n}\right)$ of various functions that need different levels of electrical consumptions. Every unit consumes a related certain electrical current ( $I_{1}$ to $I_{n}$ ) activated by the relevant virtual switches (S1 to $S_{n}$ ) respectively to activate a selected power unit according to a programmed modes of operation as shown in the Fig. 1. A setoff these internal units operate to achieve a certain operational mode. However, the basic operated units as soon as the equipment switching on are almost the internal power supply, control boards, and the display unit. These basic components consume a primary electric current. By measuring and analyzing the electrical current consumption of internal units, it is possible to know their operational status.

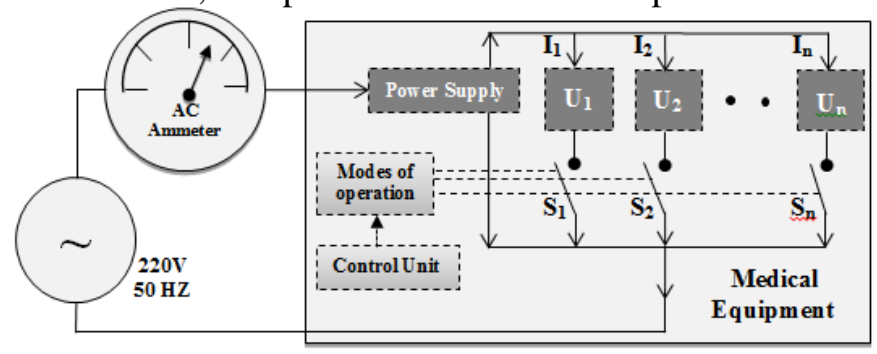

Fig. 1. Mode of Operations Methodology Detection by Electrical Consumption

Every mode consumes a certain electrical current, it can changing its values with time according to the components type of internal units. Thus, the total electric current consumed ( $\left.\mathrm{I}_{\text {Total }}\right)$ by equipment is calculated as in equation (2).

$$
\mathbf{I}_{\text {Total }}=\sum_{\text {unit }=1}^{n} \mathbf{I}_{\text {unit }}
$$

Where: $\mathrm{I}_{1}, \mathrm{I}_{2}, . ., \mathrm{I}_{\mathrm{n}}$ represent the consumed electrical current in each unit. Consequently, the consumed electric current for some mode $\left(I_{\text {mode }}\right)$ is not equal the others modes as in equation (3).

$$
I_{\text {mode }_{1}} \neq I_{\text {mode }_{2}} \neq . . \neq I_{\text {mode }_{n}} \leq \mathbf{I}_{\mathrm{Max}}
$$

Where, $\mathbf{I}_{\text {Max }}$ is the maximum electric current consumed?

In this paper, the measured Alternative electrical Current (AC) consumed by medical equipment is calculated via Root Mean Square (RMS). A measuring point of the alternative electric current must be indirect and external of the monitored medical equipment to ensure its safety and to avoid interrupting or deviating its functions.

\section{Proposed system}

The proposed system monitors and stores the consumed electric current of medical equipment by the digital ampere meter method model UT61E [25]. Fig. 2 shows the connections of the digital ampere meter in series with a monitored medical equipment and main electric power. UT61E transfer measured data through a USB (universal serial bus), an isolated RS232 connector and RS232/USB inverter to a PC to be analyzed. 


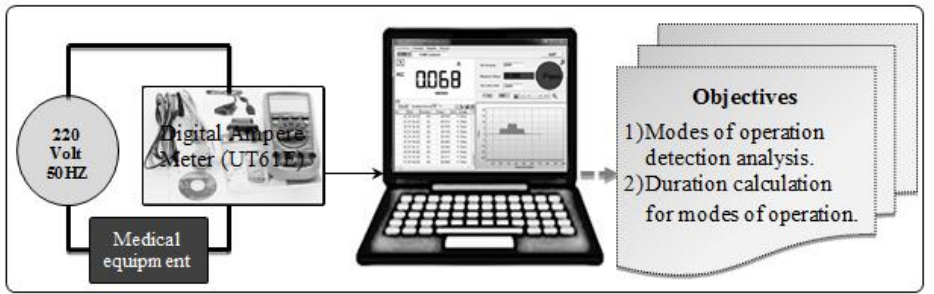

Fig. 2. The Block Diagram and Objectives of the proposed method and its validation system

\section{Experiments and results}

The tested medical equipment has single-phase electric power supply with electric current consumption less than 10 Amperes. The experiments are repeated several times to ensure precise and reliable results. The medical equipment components that consume basic electric currents during its operation such as the main boards, the power supply, the display, etc., will be referred to as the basic units, which will contribute to all modes of operation. For all the experiments, a specific mode of operation for medical equipment is selected by measuring, storing and plotting the electrical current (RMS) as a function of time. Fig. 3 shows the details of the two stages and their results.

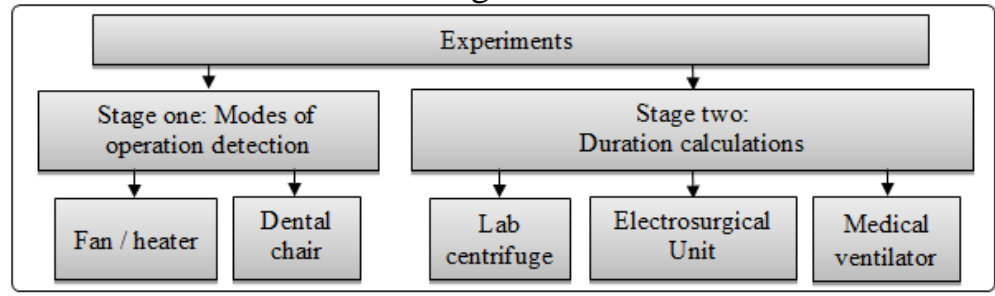

Fig. 3. Monitored equipment

\subsection{Stage one: monitoring patterns of consumption}

In this phase, the target is identifying the modes of operation through the ESA. This is applied for two equipment; a fan/heater and a dental chair.

\subsubsection{Fan/Heater}

The operation for Fan/Heater equipment is close to the manual infant warmer. The model is EHQ-2300, and the manufacturer is Elekta - China. The functioning parts in addition to the basic components are a fan motor, a half power heater, and a full power heater. These internal units consumes different amount of electric current during operation. The modes of operation are easy to detect. Fig. 4 shows different modes of operation from marker (1) to marker (5).

(1) Power off.

(2) Transient to Steady state (fan is on value).

(3) Transient to Steady state (fan \&half power heater are on).

(4) Transient to Steady state (fan \& full power heater are on).

(5) Shutdown. 
E. E. Naguib and S. A. Fawzi, A novel technique to monitor medical equipment modes of .........

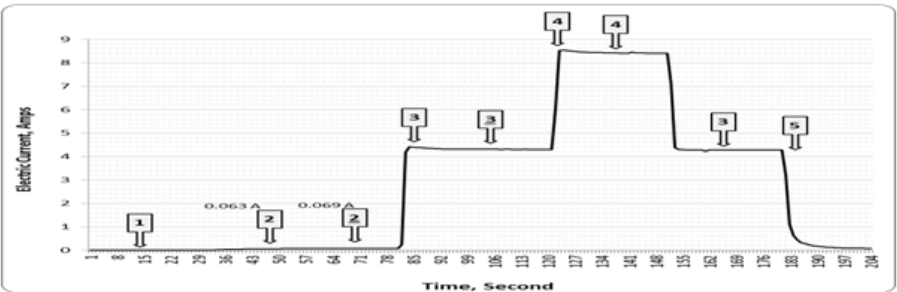

Fig. 4. Electric current consumption pattern for different modes of operation of Fan / Heater

\subsubsection{Dental chair}

The model is (1021) manufactured by Adec - USA. This model functioning parts are an Up/Down motor, a tilt motor, a light source in addition to the basic components such as electronic boards and display. Fig. 5 shows the different modes of operation from marker (1) to marker (7).

(1) Power off.

(2) Steady state operation (the basic units are on).

(3) Light source (lamp is on).

(4) Chair up (up-down motor is on).

(5) Chair down (up-down motor is on).

(6) Chair tilt (tilting motor is on).

(7) Shutdown.

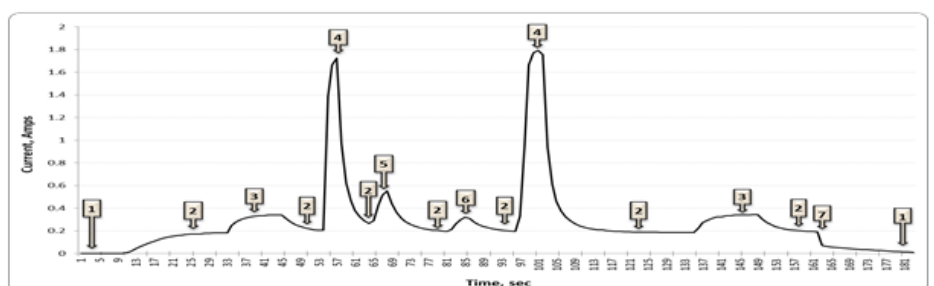

Fig. 5. Electric current consumption pattern for different modes of operation of Dental chair

\subsection{Stage two: evaluating the duration of modes of operation}

It is usually required to quantify the duration of each mode of operation obtain quantitative parameters such as availability and reliability. Modes of operation of a laboratory centrifuge, an electrosurgical unit and a medical ventilator were investigated in this stage.

In some cases, the electrical consumption values of some modes can intersect with other modes of operation especially during the transient time resulting difficulty to detect the correct modes of operation. This problem will generate an error in calculation of durations for operating mode. This problem is due to a starting-up huge values from transient impulses during switching-on causing an interference between amplitude modes of each region which separating by the electric current amplitude discriminator lines between levels of operating modes. Thus, it is required to reduce this error type by using some useful technique.

\subsubsection{Laboratory centrifuge}

The model of the laboratory centrifuge is Rotofix 32 A, and the manufacturer is Hettich [26]. The functioning parts of this model are a Motor with variable speed, in addition to the basic main units. These results were obtained while the equipment is operating without internal loads. 


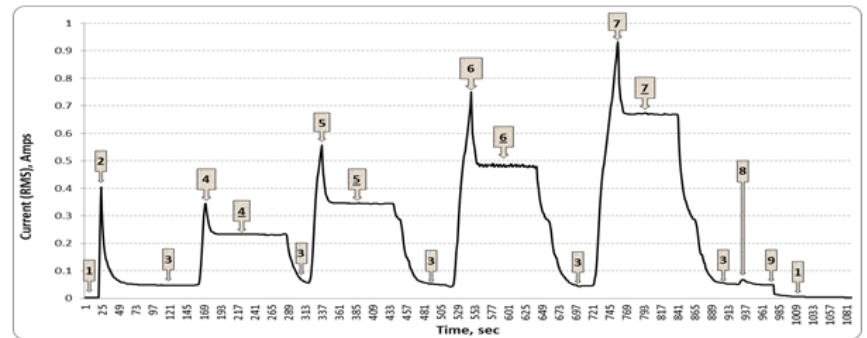

Fig. 6. Electric current consumption pattern for different modes of operation of Centrifuge

The laboratory centrifuge was monitored for duration of 1088 seconds as shown in Fig.6. The operation pattern starts with marker (1) end ends with marker (9) where the underlined marker represents the transient/startup and the other marker represents steady state.

(1) Power off.

(2) Startup (the basic units in transient state are on).

(3) Standby operation (the basic units are on in steady state).

(4) Startup and Steady state (motor at 1000 RPM is on).

(5) Startup and Steady state (motor at 2000 RPM \& basic units are on).

(6) Startup and Steady state (motor at 3000 RPM \& basic units are on).

(7) Startup and Steady state (motor at 4000 RPM \& basic units are on).

(8) Impulse (motor at short time running by key \&main units are on).

(9) Shutdown.

The intersected impulses problem of neighbor operating modes during the transient time that producing an interference error between modes of regions is resulting an error in calculation of durations for operating modes. A median filter may be used to overcome this situation.

The Median function is an important technique to reduce an error of modes calculation due to the switching of internal components inside a device which causing overlapping between consumption values during transient time of resulting impulses.

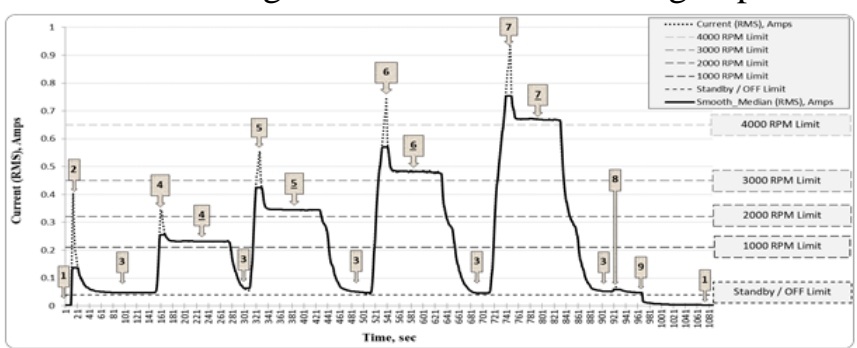

Fig.7. Electric current consumption pattern for different modes of operation of the centrifuge before and after smoothing

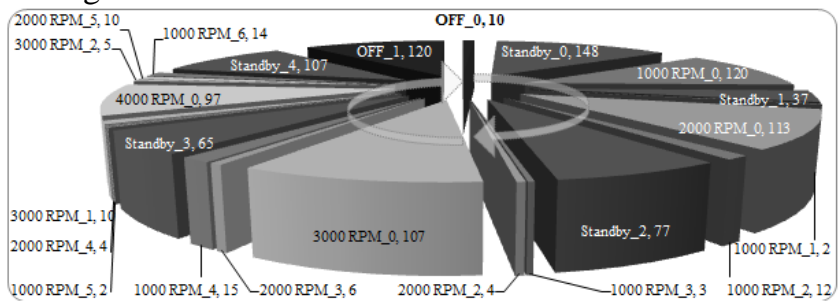

Fig. 8. Successive Modes of Operation of the centrifuge 
E. E. Naguib and S. A. Fawzi, A novel technique to monitor medical equipment modes of .........

The modified pattern is shown in Fig.7, with the doted curve represents original graph while the solid one represents the pattern after applying the Median function. The pie chart has shown in Fig. 8represents the sequence of the different modes of operation throughout certain duration while Fig.9 shows the overall duration for each mode of operation and their percentages.

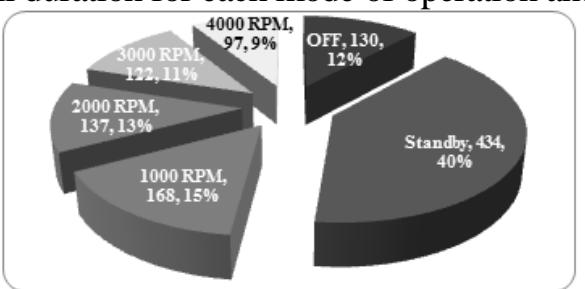

Fig. 9. Overall duration of each mode of Operation

\subsubsection{Electrosurgical unit}

Considering an electrosurgical unit, its model is (ME 102), and the manufacturer is KLS Martin. The functioning parts of this model are the power elements for cut \& coagulation in addition to the basic main units. The electrosurgical unit has two basic modes of operation, cut or coagulation with different power levels depending on each case.

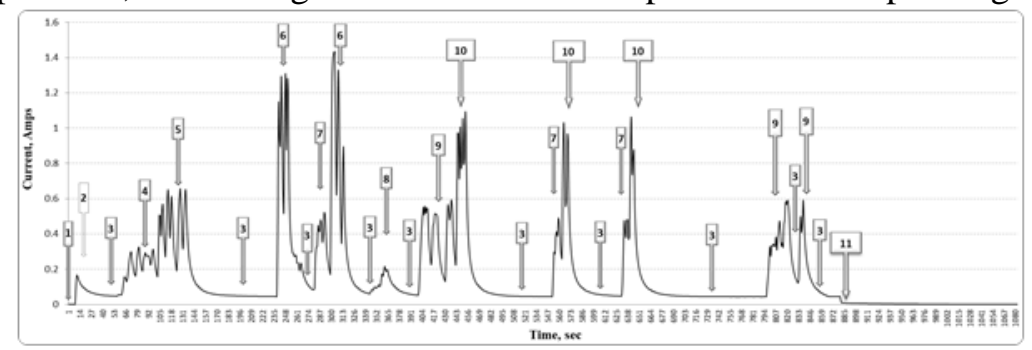

Fig. 10. Electric current consumption pattern for different modes of operation of Electrosurgical Unit

In Fig.10, different levels of both cut and coagulation were detected, as follows:

(1) Power off.

(2) Startup (basic units are in transient on state).

(3) Standby operation (basic units are in steady on state).

(4) Coagulation-3(coagulation driver at level-3 \& basic units are on).

(5) Cut-4(cut driver at level-4 \&basic units are on).

(6) Cut-8(cut driver at level-8 \&basic units are on).

(7) Coagulation-6(coagulation driver at level-6 \& basic units are on).

(8) Coagulation-2(coagulation driver at level-2 \&basic units are on).

(9) Coagulation-8 (coagulation driver at level- $8 \&$ basic units are on).

(10) Cut-6 (cut driver at level-6 \& basic units are on).

(11) Shutdown.

Moreover, the recorded electric current values of the equipment modes exceed its level due to the transient time. These values are oscillating due to both a user operating technique and different tissues' resistance types. The data processed the Median filter is useful tool to overcome the problem of overlapping the consumption values due to the spikes during the transient period of the monitored components power switching. 


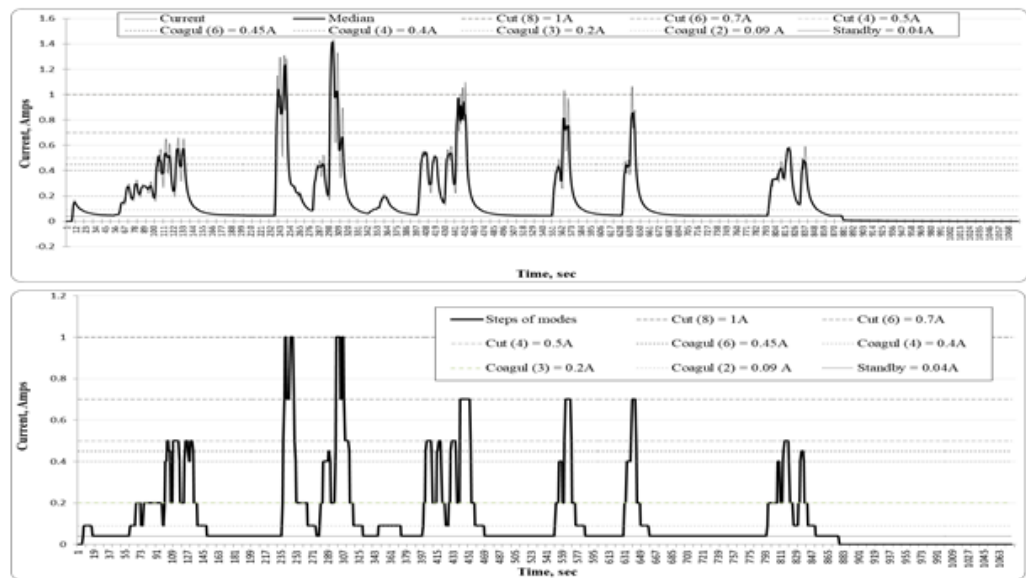

Fig. 11. Curve classification of the electrosurgical unit before and after smoothing

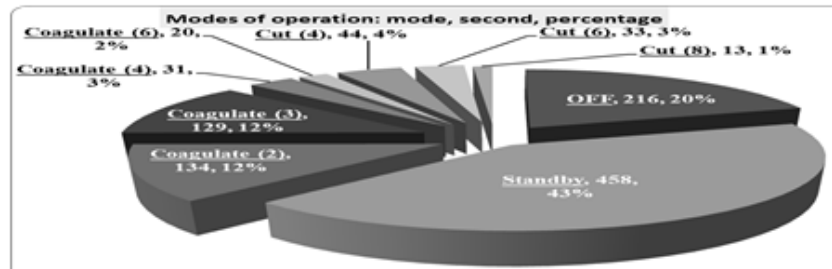

Fig.12. Duty modes of operation for Electrosurgical Unit

The dotted curve before Median function and the solid curve Median function are showing in the upper graph of Fig.11, while the lower graph represents the modified curve after classification. The pie chart in Fig. 12 shows the different modes of operation.

\subsubsection{Medical ventilator}

The model name of this equipment is Adult star 2000 and the manufacturer is Infra sonic. The functioning monitored parts in this model are a Respiration valve, SIMV valve, an Air compressor, and other main units. The main other units inside this medical equipment are compound components contains a power supply, an electronic boards, and a display.

The medical ventilator is life support equipment. It is adjustable to react with a patient case by adjusting both an inhalation rate and an exhalation rate to get the ratio between them and the respiration rate. It has a built-in internal air compressor that generates a compressed air to substitute a lack of compressed air when there is no local medical gases system is available. In this case, the consumed electric current for a respiration have two levels; low level during Assist/Control (AC) mode and high level during Synchronized Intermittent Mandatory Ventilation (SIMV) mode [27].

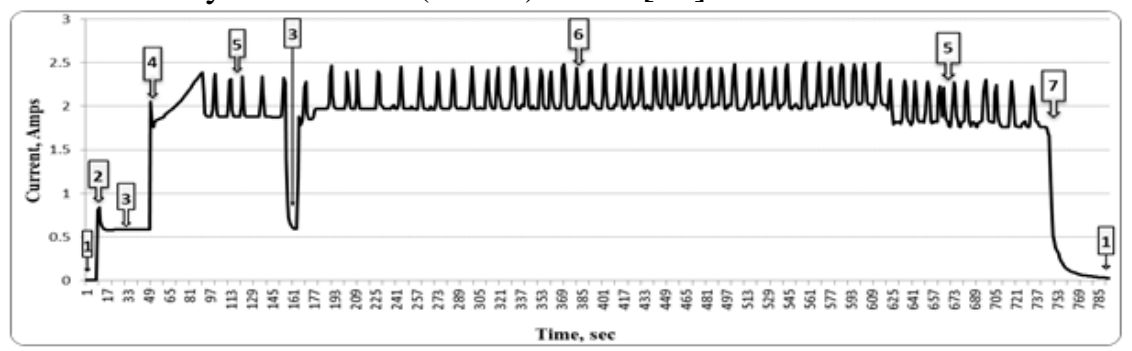

Fig. 13. Electric current consumption pattern for different modes of operation of Ventilator 
E. E. Naguib and S. A. Fawzi, A novel technique to monitor medical equipment modes of .........

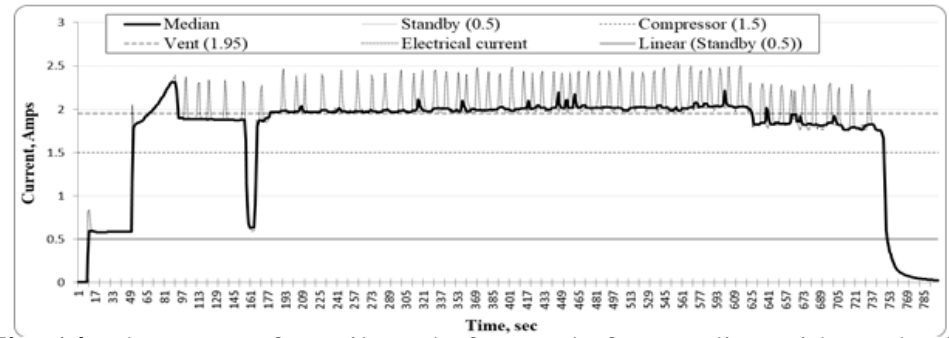

Fig. 14. The curve of ventilator before and after Median with modes levels

In Fig.13, many modes of operation for a respiration rate and an air compressor detected during certain time from marker (1) to marker (7).

(1) Power off.

(2) Startup (basic units are in transient state).

(3) Standby (basic units are in on steady state).

(4) Compressor is running (compressor \&basic units are on).

(5) A/C mode (compressor \& respiration valve \& basic units are on).

(6) SIMV mode (compressor \& respiration valve \& SIMV valve \& basic units are on).

(7) Shutdown.

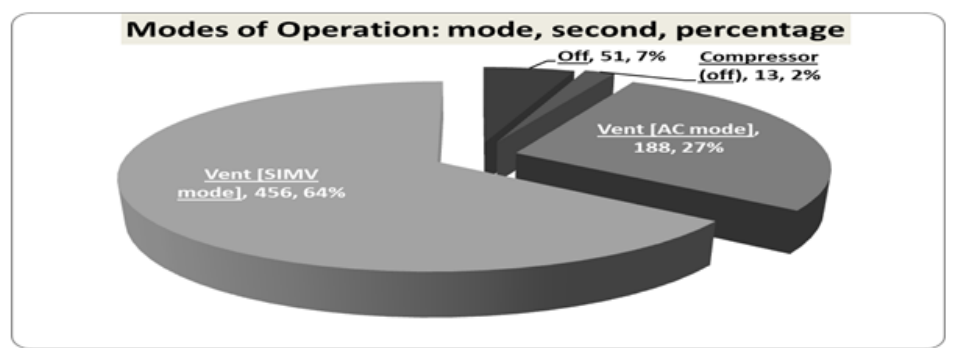

Fig. 15. Duty modes of operation for ventilator during certain time

By smoothing (Median function) the dotted curve for about twenty successive items of data, the output-soled curve after filtration produces roughly two electric current levels. These two levels have roughly not respiration pulses. The two levels reflect the different respiration rates as shown in Fig.14 that shows the signal before and after filtration. Fig.15 gives the complete modes of operation for the medical ventilator equipment.

\section{Discussion}

Most of the internal components inside medical equipment are working in regulated and isolated DC voltage than main AC voltage via switching mode power supply (SMPS) where almost equipment requires power supplies with size compact and effective in power conversion by pulse width modulation (PWM) [28].

The monitoring type for medical equipment is external, isolated, and indirect. Therefore, it does not have any side effects during operation of monitored medical equipment and it obeys the standards of international medical equipment. These experiments proved that the amplitude of a consumed electrical current by the monitored medical equipment is important factor to deduce its operating modes. The high power consumption components inside a medical equipment such as motors, electric valves, electric heaters, or electric drivers improves the operating modes detection compared by the low power components internal it. The number of monitored functions changes from a 
medical equipment to other according to its type and model. Every equipment have an individual functions, so it is important to study every case alone except the equipment with identical brand name and model.

These experiments executed by the ammeter model UT61 to monitor the modes operation to detect it by comparison of the amplitude of electric current consumption only. The fan/heater operating modes detection case is easier than the dental chair where the last one is more complex than first one according to the complexity of equipment. It is notable that electric current consumption during "chair go up" operation is greater than "chair go down" due to gravitational force.

The experiments in phase two had done to calculate the modes of operation periods during a certain time for centrifuge, electrosurgical unit, and medical ventilator.

In the centrifuge, many modes of operation like standby, and four values of Revolutions per Minutes (1000, 2000, 3000, and 4000 RPM) detected by comparing different electric consumption amplitudes for each. These modes of operations detection are depending on an angular rotational (RPM) of the internal motor of the laboratory centrifuge. These detected operating modes duration are useful to monitor not only the usability of the equipment but also to calculate variation of running stresses on internal motor that is an important factor to find the maintainability strategy by a decision maker.

The detected modes for the electrosurgical unit are depending on not only a used operating modes but a user's sensitivity also. Therefore, it is more difficult to detect operating modes than before.

The detected duration modes in the ventilator are useful to follow the patient effective therapy related to the used ventilation mode. As a result, a pie chart concludes modes of operation of some equipment during certain time. This chart is useful and important to understand availability, usability, maintainability, internal power components stresses of monitored devise, evaluation of life cycle, and scrapping.

By sending the data of each monitored medical equipment inside hospital to the hospital server, it is possible to monitor the most medical equipment, analyses the related results, and merging it with hospital information system (HIS) to save it in hospital data base and sending it to a decision maker when request.

This paper is just a fundamental stone of an integrated system. This method requires many improvements to grow up as an integrated system. The first step to upgrade this method to an integrated system is designing an appropriate dedicated embedded system instead of the used electric current meter gauge. This paper is limited also to five different types of medical equipment analyzed in time domain only to prove concept of study. Hence, the more and more studying is required to cover most types of the existed market share medical equipment and adding extra techniques to analyze electrical signature.

\section{Conclusions}

The performed experiments through two stages describe a new successful technique to detect the modes of operation of medical equipment through external reading for power consumption with no influence on the equipment operation.

This study proved that the electric current consumption rate from a main power plug especially by power components such as motors, electric valves, electric heaters or electric 
E. E. Naguib and S. A. Fawzi, A novel technique to monitor medical equipment modes of .........

drivers detects a certain operating mode is an effective tool to monitor the medical equipment operation.

Applying the technique on different medical equipment showed that each one has a characteristic curve of current consumption for each mode and each type of equipment such as electrical signature. This curve is specific only for that equipment and may vary with the model, manufacturer and mode of operation. This monitoring strategy has no effect on the equipment operation and satisfies the international standards of an indirect power consumption measurement.

The monitoring of medical equipment usability durations for each operating mode is important information for the decision maker to record the effective running time and saving it to its database.

\section{REFERENCES}

[1] Williams, P. A., \& Woodward, A. J., "Cybersecurity vulnerabilities in medical devices: a complex environment and multifaceted problem", Medical Devices (Auckland, NZ), 8, 305, 2015.

[2] World Health Organization, "Health technology assessment of medical equipment", 2011.

[3] World Health Organization, "Medical equipment maintenance program overview", 2011.

[4] Ibrahim, A. M., \&Dimick, J. B., "Monitoring medical devices: missed warning signs within existing data", Jama, 318(4), 327-328, 2017.

[5] Rosow, E., \& Adam, J.,"Real-Time Executive Dashboards and Virtual Instrumentation: Solutions for Health", Clinical engineering handbook, 476, 2004.

[6] Bayati, M., Braverman, M., Gillam, M., Mack, K. M., Ruiz, G., Smith, M. S., \& Horvitz, E.,"Data-driven decisions for reducing readmissions for heart failure: General methodology and case study",PloS one, 9(10), e109264, 2014.

[7] YAng, Y., Lu, Z., Luo, X., Ge, Z., \& Qian, Y., "Mean failure mass and mean failure repair time: parameters linking reliability, maintainability and supportability", EksploatacjaiNiezawodność, 16(2), 2014.

[8] Tee, K. F., \&Ekpiwhre, E., "Reliability-based preventive maintenance strategies of road junction systems", International Journal of Quality \& Reliability Management, 2019.

[9] Mokhtar, A. A., Misren, M. M., Muhammad, M., \&Hussin, H.,"RAM analysis of crude oil transfer pumps using dominant failure mode", 2006.

[10] Mohammadi, M., Rai, P., \& Gupta, S., "Performance measurement of mining equipment", International Journal of Emerging Technology and Advanced Engineering, 5(7), 240-248, 2015.

[11] Barbarosoglu, B. V., \&Arditi, D., "A System for Early Detection of Maintainability Issues Using BIM" In Advances in Informatics and Computing in Civil and Construction Engineering (pp. 335-341). Springer, Cham, 2019.

[12] Epstein, A. L., \& Harding, G. H. "Risk Management in Selected High-Risk Hospital Departments", Risk Management in Healthcare Institutions: Limiting Liability and Enhancing Care, 301, 2012.

[13] Baretich, M., "Equipment control and asset management", Clinical engineering handbook, 1, 122, 2004.

[14] Faiz, R. B., \& Edirisinghe, E. A, "Decision making for predictive maintenance in asset information management", Interdisciplinary Journal of Information, Knowledge, and Management, 4(1), 23-36, 2009.

[15] Mathew, A. D., Zhang, S., Ma, L., Earle, T., \& Hargreaves, D. J.,"Reducing maintenance cost through effective prediction analysis and process integration", Advances in vibration engineering, 5(2), 87-96, 2006. 
[16] Hameed, Z., Hong, Y. S., Cho, Y. M., Ahn, S. H., \& Song, C. K., “Condition monitoring and fault detection of wind turbines and related algorithms: A review", Renewable and Sustainable energy reviews, 13(1), 1-39, 2009.

[17] Blumberg, D. F., "Advanced diagnostics and artificial intelligence", In Clinical engineering handbook (pp. 464-475), Academic Press, 2004.

[18] Liu, X., Lodewijks, G., \& Pang, Y., "Intelligent Maintenance of Large-scale Belt Conveyor Idler Rolls: State-of-the-art and Opportunities", TU Delft, 1-5, 2014.

[19] Salomon, C. P., Santana, W. C., Lambert-Torres, G., da Silva, L. E. B., Bonaldi, E. L., de Oliveira, L. E. D. L., ... \& Lopes, M. A. A., "Discrimination of synchronous machines rotor faults in electrical signature analysis based on symmetrical components", IEEE Transactions on Industry Applications, 53(3), 3146-3155, 2016.

[20] Shahriar, M. R., Borghesani, P., \& Tan, A. C., "Electrical signature analysis-based detection of external bearing faults in electromechanical drivetrains", IEEE Transactions on Industrial Electronics, 65(7), 5941-5950, 2017.

[21] Zarabi, S., Fernandes, E., Rua, I., Salehian, A., Debéda, H., Nairn, D., \& Wei, L., “Design and development of a self-contained and non-invasive integrated system for electricity monitoring applications", In 2017 IEEE 12th International Conference on ASIC (ASICON) (pp. 1125-1128). IEEE, 2017.

[22] Sa, J., Choi, Y., Chung, Y., Kim, H. Y., Park, D., \& Yoon, S., "Replacement condition detection of railway point machines using an electric current sensor", Sensors, 17(2), 263, 2017.

[23] Ishida, Y. "Current measurement device, control method for same, recording medium, and power measurement device", U.S. Patent No. 9,921,247. 20 Mar. 2018.

[24] Discenzo, F., Unsworth, P., Vetcha, S. B., Loparo, K. A., Dister, C. J., \&Tomkin, E. J., U.S. Patent No. 7,539,549. Washington, DC: U.S. Patent and Trademark Office, 2009.

[25] Uni-t UT61E Manuals, https://www.manualslib.com/products/Uni-T-Ut61e3834617.html,(Accessed: 23/4/2019).

[26] Manual ROTOFIX $\quad 32$ A, www.hettichlab.com/downloadcenter/Products/Manuals/ROTOFIX_32_A/AB1206DEENFR IT.pdf,(Accessed: 28/4/2019).

[27] Features of Infrasonics Adult Star 2000 Ventilator,https://avantehs.com/p/infrasonics-adultstar-2000-ventilator/1136,(Accessed: 30/4/2019).

[28] Lu, H. H., \& Chou, K. H., "Control methods and switching mode power supplies with improved dynamic response and reduced switching loss." ,U.S. Patent Application No. $15 / 692,562,2018$.

\section{تقنية جليدة ترصد أوضاع تثنيل الأجهزة الطبية}

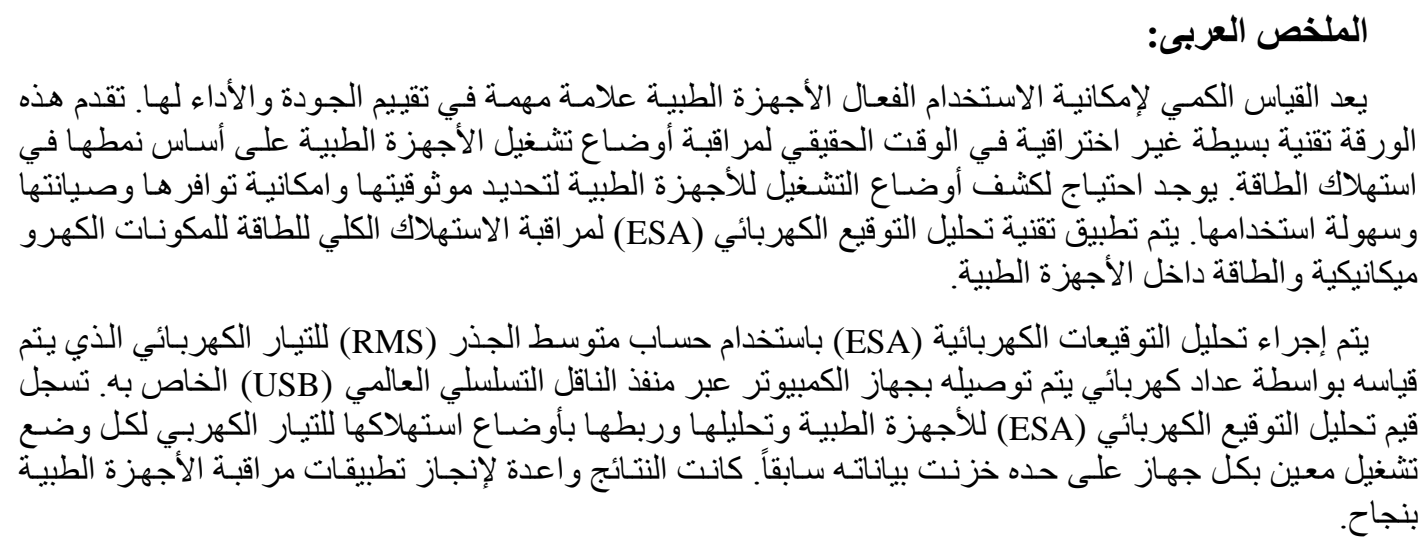

